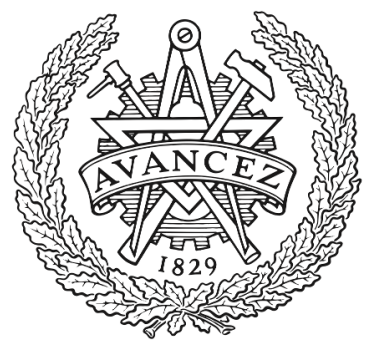

CHALMERS

UNIVERSITY OF TECHNOLOGY

\title{
Self-calibration algorithms for microwave hyperthermia antenna arrays
}

Downloaded from: https://research.chalmers.se, 2023-04-26 08:51 UTC

Citation for the original published paper (version of record):

Zanoli, M., Persson, M., Dobsicek Trefna, H. (2018). Self-calibration algorithms for microwave hyperthermia antenna arrays. IET Conference Publications, 2018(CP741).

http://dx.doi.org/10.1049/cp.2018.0933

N.B. When citing this work, cite the original published paper. 


\title{
Self-calibration algorithms for microwave hyperthermia antenna arrays
}

\author{
Massimiliano Zanoli, Mikael Persson, Hana Dobšíček Trefná \\ Dept. Electrical Engineering, Chalmers University of Technology, Gothenburg, SE \\ zanoli@chalmers.se
}

\begin{abstract}
In deep microwave hyperthermia (MW-HT), antenna arrays are used to generate an interference pattern which focuses energy in the tumor location. These arrays are subject to a number of disturbances which must be compensated for through calibration. This paper proposes and analyzes a pair of self-calibration algorithms, i.e. calibration procedures which rely only on S-matrix measurements of the N-port array applicator device, avoiding the need for external references and making real-time in-treatment calibration possible. Two algorithms are analyzed by means of simulations and experiments in terms of reliability and sensitivity to different kinds of disturbances. The results show that one of two implemented algorithms can converge to the same calibration values obtained when using an external calibration reference (monopole antenna).
\end{abstract}

Index Terms-microwave, hyperthermia, array, selfcalibration.

\section{INTRODUCTION}

Microwave hyperthermia (MW-HT) is a well known adjuvant modality for cancer treatment [1]. In MW-HT, energy is deposited into the tumor in a non-invasive manner by radiating antennas. When the target is located deeply within the patient body, it is necessary to use antenna arrays in order to reach satisfying volume coverage and treatment efficacy (Fig. 1, left). By steering the amplitudes and phases of the array elements it is possible to shape the wave propagation so that constructive interference generates a focus in the tumor location, while sparing the surrounding healthy tissues [2]. These arrays represent sophisticated multi-port devices and are subject to various types of small disturbances which cannot be modeled during treatment planning, such as: different cable lengths, array manufacturing tolerances, antenna location offsets, patient misplacement and irregular folding of the so called water bolus collar used for dielectric matching and skin cool down (Fig. 1, right).

When operating at frequencies around $100 \mathrm{MHz}$ with a typical system, the phase errors introduced by such disturbances are reduced and can be sufficiently compensated for by a oneoff single channel calibration of the system. On the other hand, the focal diameter achieved when treating muscular tissues at this frequency is around $13 \mathrm{~cm}$, which seriously limits the tumor heating capability due to constraints on the surrounding healthy tissues. It is therefore highly desirable to adopt shorter wavelengths as they allow for a smaller and more accurate focusing to be achieved. When selecting higher operating frequencies however, the amount of amplitude distortion and

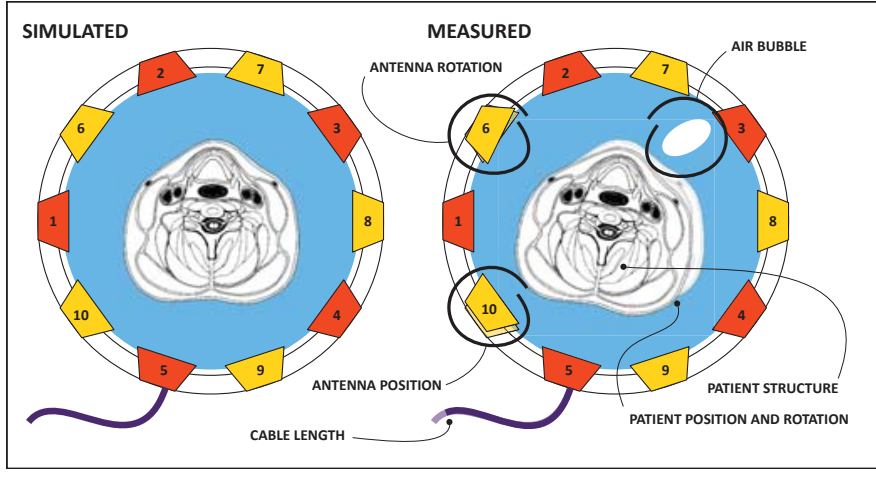

Fig. 1. Ideal array, water bolus and patient setup for neck MW-HT (left), and scheme for considered possible mismatches (right). Red and yellow colors indicate antennas on different rings.

phase misplacement due to disturbances can be such as to alter the predicted scattering pattern and prevent focus formation, affecting the efficacy and safety of the treatment. Furthermore, as the wave propagation becomes more sensible to small geometrical mismatches, any attempt of pre-calibrating the whole array system with an external known reference (such as a monopole antenna) right before the treatment session might be invalidated by subsequent patient insertion.

All these issues could be addressed with the use of a selfcalibration (SC) technique. SC is a broad concept in system engineering, which aims at overcoming the necessity for an external calibration reference. In MW-HT, this means that the procedure can be carried out while the patient is in position, thus providing real-time compensation for disturbances throughout the whole treatment session. Since the implementation of the SC technique is strictly dependent on the particular system design, custom SC algorithms have to be implemented for the specific HT problem. This paper describes two potential solutions to the phase error aspect of the SC problem. This is done by exploiting the measurements of the $S$ matrix for the whole array device as well as simulated S-parameters from corresponding virtual models of the applicator.

An analysis of the algorithm sensitivity to specific disturbances is carried out by means of simulations, using the commercially available software CST Studio (Dassault Systmes). Finally, the proof-of-concept is demonstrated experimentally using the Head \& Neck MW-HT prototype system developed at Chalmers University of Technology [3]. 


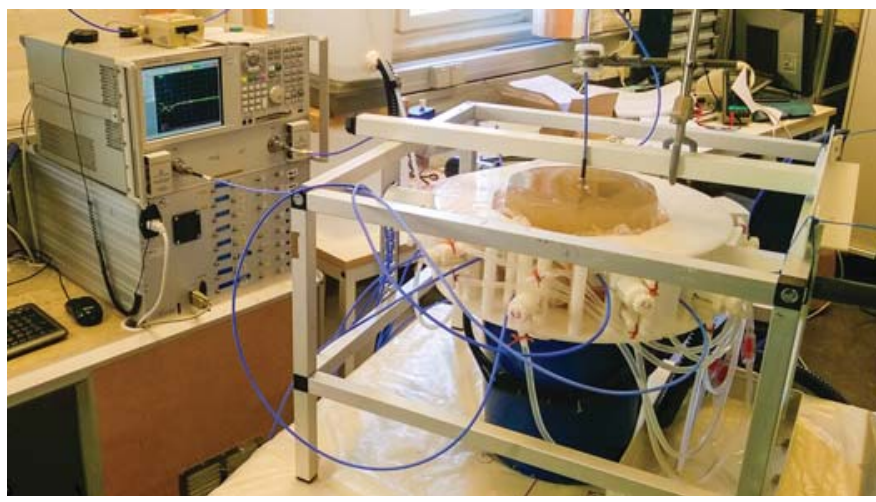

Fig. 2. Experimental setup. The external reference (monopole antenna) is visible on top of the muscle-mimicking decagonal phantom.

\section{Methods}

Given a specific operating frequency for the hyperthermia system, the scope of the self-calibration (SC) is to obtain a set of phase and amplitude correction values for each antenna channel that compensate for disturbances at the applicator side, thus enabling proper focus steering during treatment. We focus on phase calibration, i.e. we aim at finding vector:

$$
\Phi=\left(\phi_{1}, \phi_{2}, \ldots, \phi_{N}\right) \quad[\mathrm{rad}]
$$

where $\phi_{n}$ is the phase compensation for channel $n$, and $N$ indicates the number of antennas in the array. Two different implementations of SC have been designed and tested:

- Triplet-Consensus Calibration (TCC) determines the exact transmission delay values experienced by antenna subsets, represented by all the possible combinations of 3 antennas. By using simulated delay values as a reference, the algorithm solves an exact system of equations for 3 unknown $\phi_{n}$ values:

$$
\left\{\begin{array}{l}
\phi_{1}+\phi_{2}=\Delta \phi_{1,2}^{\mathrm{M}}-\Delta \phi_{1,2}^{\mathrm{S}} \\
\phi_{1}+\phi_{3}=\Delta \phi_{1,3}^{\mathrm{M}}-\Delta \phi_{1,3}^{\mathrm{S}} \\
\phi_{2}+\phi_{3}=\Delta \phi_{2,3}^{\mathrm{M}}-\Delta \phi_{2,3}^{\mathrm{S}}
\end{array}\right.
$$

where $\Delta \phi_{i, j}$ is the transmission delay (as phase offset) of the path going from antenna $i$ to antenna $j$ ( $S$ is simulated and $M$ is measured). As different subsets will yield different solutions, a consensus step is subsequently performed in order to build a complete set of $N$ values. Two subsets are regarded as agreeing if the calibration values returned for their common antennas are equivalent within a threshold $T H_{\phi}$.

- Jacobian-Optimization Calibration (JOC) aims at minimizing the difference between simulated and measured antenna-to-antenna path delays by means of quadratic optimization, where the optimized parameters are the unknown $\Phi$ delays experienced by each antenna:

$$
C(\Phi)=\sum_{i=1}^{N} \sum_{j=1}^{N} w_{i, j}\left(\Delta \phi_{i, j}^{\mathrm{M}}\left(\phi_{i}, \phi_{j}\right)-\Delta \phi_{i, j}^{\mathrm{S}}\right)^{2}
$$

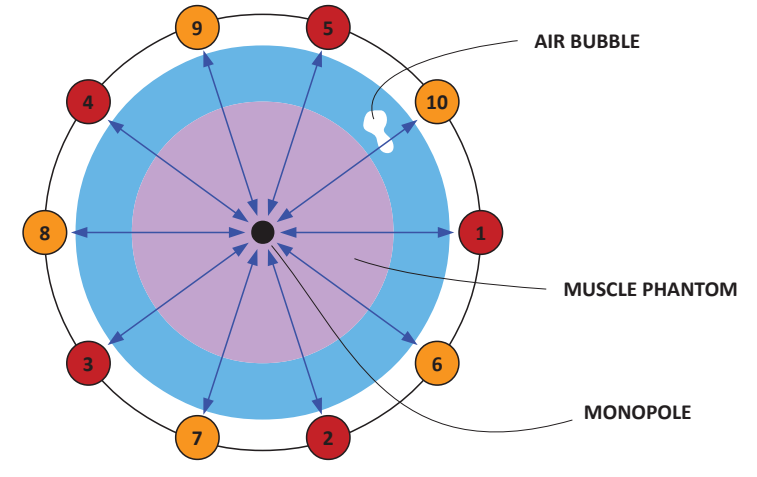

Fig. 3. Schematic for monopole calibration. Blue arrows indicate antenna-tomonopole transmission paths.

where $C$ denotes the cost function and $w_{i, j}$ is the weight of the $(i, j)$ transmission path, which are in turn computed according to the shape of the antenna lobes.

Both algorithms rely on simulated path delay values as a reference for calibration, and therefore require a simulation step to be performed before they can be run. This is usually not a problem in MW-HT, where the so-called treatment planning step makes already heavy use of simulations for determining the best amplitude and phase steering parameters for proper tumor focusing [2]. The output of these include the full Smatrix relative to the ideal $3 \mathrm{D}$ model of the applicator and patient system needed by the SC algorithms.

\section{A. Performance assessment}

Quantitative evaluation of the performances of the SC algorithms has been carried out by comparing their output with the calibration values obtained using an external monopole or dipole antenna (Fig. 3). When the contents of the array are perfectly symmetrical (for instance, when a cylindrical phantom is used), a monopole antenna placed in the center should receive with the same delay from all the other antennas. If a disturbance is introduced, e.g. a slight misplacement of one antenna, the monopole senses a phase offset and the channel feed has to be compensated accordingly.

The SC algorithms return phase compensation values by looking at the S-parameter matrix measured in presence of the disturbance. By comparing self and external calibration values, and assuming the latter to be the correct ones, the SC error for each channel is obtained:

$$
\tilde{\Phi}=\left|\Phi^{\mathrm{SC}}-\Phi^{\mathrm{EX}}\right|
$$

where $E X$ indicates the external calibration (monopole or dipole). Average $(A V G)$ and maximum $(M A X)$ errors can then be extracted from this error vector for different amounts of disturbance. 


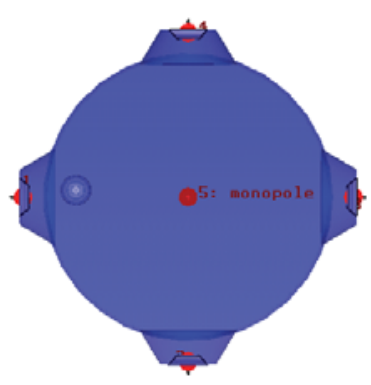

(a)

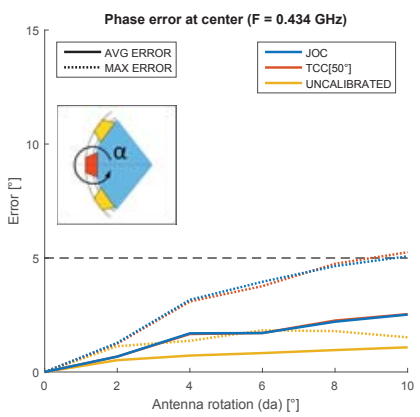

(e)

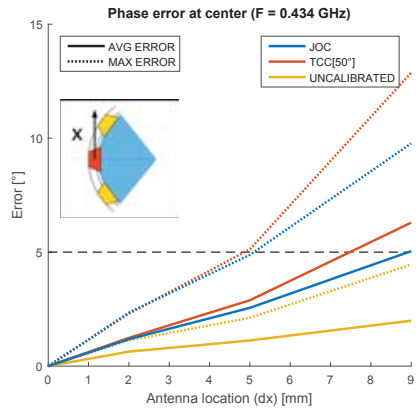

(b)

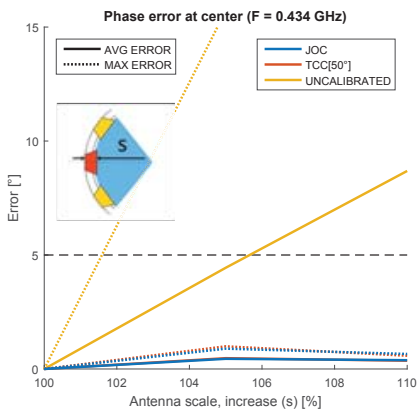

(f)

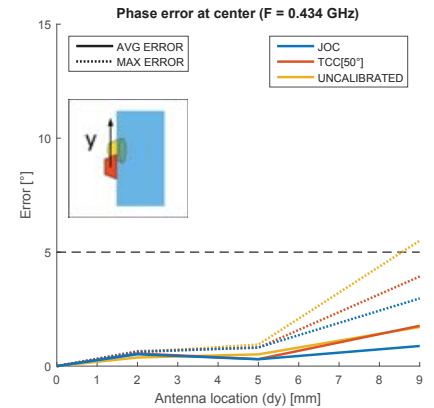

(c)

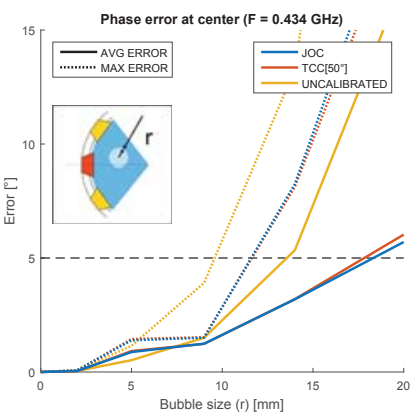

(g)

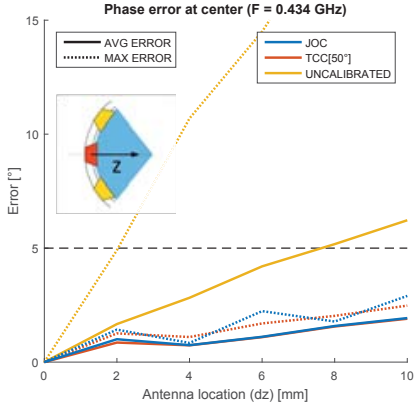

(d)

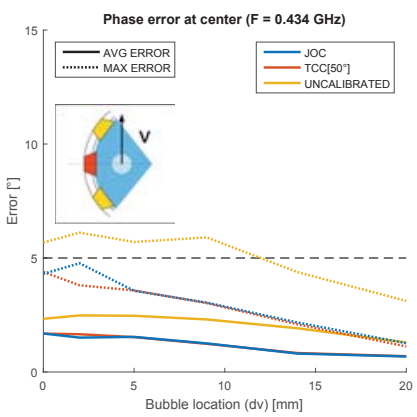

(h)

Fig. 4. Self-calibration sensitivity analysis results using a 4 antenna array model (a). Maximum and average channel phase error with respect to the phase calibration values yielded by external calibration, i.e. monopole antenna in the applicator center. JOC and TCC (with $50^{\circ}$ consensus threshold) algorithm results are reported, together with non-calibrated values. Black dashed axis denotes the $5^{\circ}$ safety limit for deep MW-HT. The inner panels report a reference scheme for the specific type of disturbance analyzed.

\section{B. Sensitivity analysis}

For the present work, the types of disturbance shown in Fig. 1 (right) have been simulated in virtual models of a 4 antenna applicator (Fig. 4a), in particular:

- Antenna mismatches, which include misplacement $(\mathrm{dx}$, $\mathrm{dy}, \mathrm{dz}$ in Fig. 4b, 4c, 4d), error in pointing direction (da, Fig. 4e) and uniform scaling factor (s, Fig. 4f).

- Air bubble size (r, Fig. 4g) and position (dv, Fig. 4h). The air bubble introduces an undesired discontinuity in the medium's impedance and models the inappropriate folding of the water bolus collar.

In a second set of sensitivity models based on an 8 antenna applicator, random amounts for the aforementioned disturbances (including scattered air bubbles) are introduced across the whole applicator, so that each antenna is subject to a certain degree of distortion in field propagation (Fig. 5, small panel). Once a model is prepared using a set of generated mismatches, the algorithms are run with different operating frequencies as target for calibration. In this way, the sensitivity of the algorithm with respect to operating frequency is assessed. The analysis is carried out for frequencies ranging from 0.3 to 1.0 $\mathrm{GHz}$, which is roughly the working bandwidth of the UWB bow-tie antennas used for this applicator [4].

\section{Experimental setup}

The 10 antenna MW-HT applicator prototype has been used for measurements on a muscle-mimicking phantom realized with algae gel, according to [5]. The phantom is decagonal and completely fills the internal applicator volume (Fig. 2). With a symmetrical setup, external calibration is possible by placing the reference in the center of the phantom. The S-matrix is measured by means of a 24-channels VNA instrument and subsequently fed to the two SC algorithms previously described.

\section{RESULTS}

\section{A. Simulation results}

The sensitivity analysis shows that among the possible antenna displacements (Fig. 4b, 4c and 4d), an offset along the $Z$ axis is the most correctly compensated for by the selfcalibration (SC) algorithms. While the antennas are usually fixed along their $X$ and $Y$ axes, movement along $Z$ is left as a degree of freedom in circular arrays, so that the array radius can be adjusted to fit the patient's anatomy. $\mathrm{dz}$ is thus a relevant type of disturbance which is correctly handled by the SC algorithms. Antenna rotation (Fig. 4e) is less effectively addressed but usually impeded during array design, and antenna size (Fig. 4f), although well compensated for, is also unlikely to happen in arrays manufactured with 


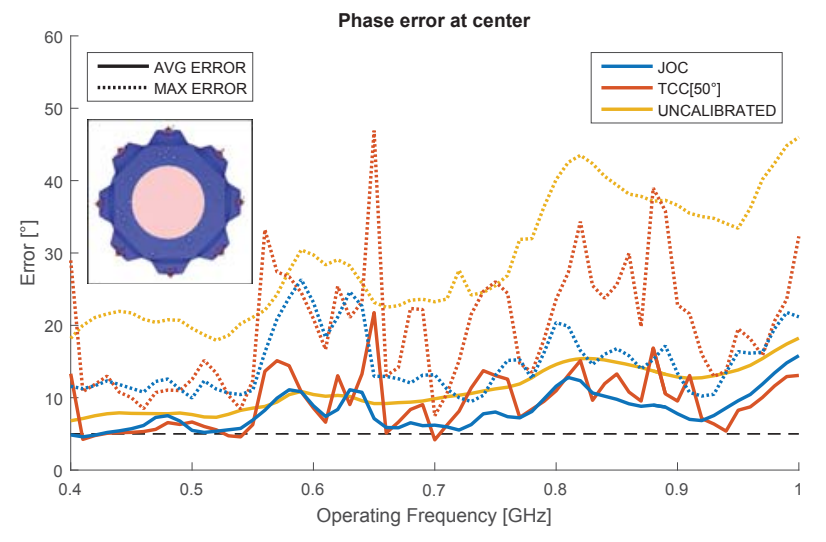

Fig. 5. Frequency sensitivity of the SC algorithms on a model with randomly distributed mismatches. Black dashed axis denotes the $5^{\circ}$ safety limit for deep MW-HT.

mechanical precision. Presence of an air bubble in the water bolus zone (Fig. 4g and $4 \mathrm{~h}$ ) is also correctly compensated, with the phase error at center always kept below $5^{\circ}$. Please note that the phase errors for a no-calibration solution $\left(\phi_{n}=0, \forall n\right)$ are also reported in the plots for comparison (UNCALIBRATED).

The frequency sensitivity results (Fig. 5) obtained on a model with exaggerated randomly generated mismatches (all those enlisted in Sec. II-C) exhibit strong resonance phenomena across the $0.3-1.0 \mathrm{GHz}$ spectrum. These might be due at least in part to the comparison with the external monopole antenna, which is subject to structural resonances in the model and might not always yield the best calibration solution for any operating frequency, especially from the point of view of the overall e-field distribution. On average however, the JOC algorithm (and less TCC) can improve the phase error by $3^{\circ}$ with respect to having no calibration.

\section{B. Experimental results}

Experimental results for JOC (Fig. 6a) show that the self and external calibration solutions converge within a narrower operating frequency bandwidth around $0.5 \mathrm{GHz}$, while TCC is not able to achieve the same performances as JOC (Fig. 6b). This proves on one hand the ability of the JOC SC algorithm to compensate for different cable lengths and geometrical mismatches in a real scenario, but also raises questions about the validity of monopole calibration when working with frequencies outside its response spectrum. Furthermore, at higher frequencies, unavoidable small errors in physical monopole placement can easily invalidate the returned calibration values.

\section{CONCLUSIONS}

This study has proven self-calibration (SC) to be a potential candidate for use in MW-HT systems. The JOC implementation of SC is the most promising among those tested, as it yields compensating phase values similar to those obtained when using an external reference antenna for calibration. The SC algorithms exhibit a rather stable sensitivity to various types of array disturbances for a satisfying range of values,

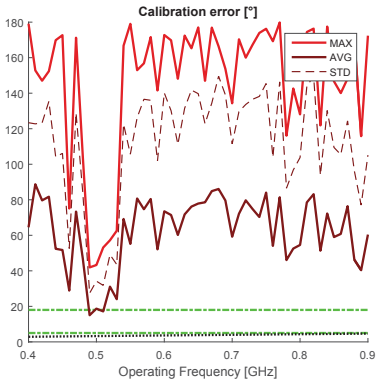

(a) JOC

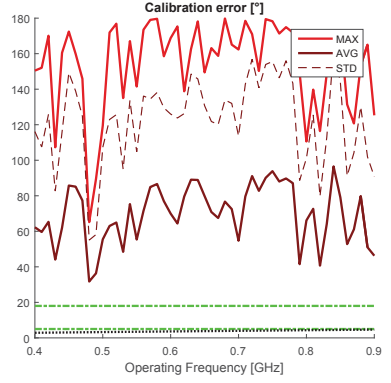

(b) TCC
Fig. 6. Frequency sensitivity analysis for physical prototype. Green dashed axis denotes $5^{\circ}$ and $18^{\circ}$ (corresponding to $5 \%$ amplitude loss) safety limits for deep MW-HT.

which correspond to the mismatch expected in a typical HT system.

The use of monopole antennas as references for performance assessment can be problematic, as these are susceptible to resonance phenomena which might invalidate the comparisons at certain frequencies. To obtain a more complete picture of the overall performances of the SC algorithms, efield simulations and thermal distribution measurements are necessary, in order to fully assess the compensation abilities of the SC algorithms.

The theoretical benefits of using self-calibration in MWHT, namely the possibility of compensating for disturbances and patient mismatches in real-time throughout the whole HT session, represent a motivation for continuing investigating the performances and limits of these algorithms. If SC methods are employed together with UWB antenna arrays, the potential increase in operating frequency might enable MW-HT technology to safely reach focal volumes half in size with respect to today's state of the art MW-HT systems.

\section{ACKNOWLEDGMENT}

This work was supported in part by VINNOVA (Swedish Government Agency for Innovation Systems) within the VINN Excellence Center ChaseON.

\section{REFERENCES}

[1] Datta, N. R., et al. "Local hyperthermia combined with radiotherapy and/or chemotherapy: Recent advances and promises for the future." Cancer treatment reviews 41.9 (2015): 742-753.

[2] Kok, H. P., et al. "Current state of the art of regional hyperthermia treatment planning: a review.” Radiation Oncology 10.1 (2015): 196.

[3] Trefná, H. D., et al. "Design of a wideband multi-channel system for time reversal hyperthermia." International Journal of Hyperthermia 28.2 (2012): 175-183.

[4] Yang, J., et al. "A novel low-profile compact directional ultra-wideband antenna: the self-grounded Bow-Tie antenna." IEEE Transactions on Antennas and Propagation 60.3 (2012): 1214-1220.

[5] Trefná, H. D., et al. "Quality assurance guidelines for superficial hyperthermia clinical trials: I. Clinical requirements." International Journal of Hyperthermia 33.4 (2017): 471-482. 